\title{
ALZHEIMER'S DISEASE - MODERNIZING CONCEPT, BIOLOGICAL DIAGNOSIS AND THERAPY
}

\author{
Harald Hampel and Maria C. Carrillo \\ Karger Publishers, Basel (Switzerland), 2012, 194 pages \\ ISBN: 978-3-8055-9802-6. Price: $56 €$
}

This is an international book which brings together the up to date knowledge of Alzheimer' disease. In the context of the worldwide epidemic of dementia the book has emerged as an expected product of American and European experts, mainly from the neurological field. It is edited by Dr. Hampel and Dr. Carrillo, prestigious authors that were able to bring together leaders in different aspects of the disease.

The book summarizes major topics related to the disease, namely the numbers of individuals affected and the global impact of this condition; the genetics, neuropathological bases and current conceptual views; the diagnostic tools, both biomarkers and modern neuroimage techniques; the new diagnostic concepts; and the pharmacological treatments. It also approaches the regulatory requirements for clinical trials and ends up with a chapter on the perspectives on Alzheimer's Disease.

While the book is not extensive and parts of the content may be somewhat controversial, for example when summarizing the epidemiological data, this text may be a good reference for the chapters covered, mainly for neurologists. Psychiatrists and neuropsychologists may miss areas considered to be relevant, including the non-cognitive, psychopathological symptoms or the psycho-social treatments. However, this volume contributes valuably by outlining basic concepts of Alzheimer's disease, reviewing the empirical background and describing new methods to approach this condition and to enable clinicians to have an up to date view of the disease, its origins and the new perspectives to advance in both the knowledge and the care of patients.

Antonio Lobo, MD

Concepción De la Cámara, MD

Miguel Ángel Quintanilla, MD

Psychiatry Service, Hospital Clínico Universitario, Zaragoza

Department of Psychiatry, Faculty of Medicine, University of Zaragoza

SPAIN 